\title{
Spectrum of quenched twisted mass lattice QCD at maximal twist
}

\author{
Abdou M. Abdel-Rehim and Randy Lewis \\ Department of Physics, University of Regina, Regina, SK, Canada, S4S 0A2 \\ R. M. Woloshyn \\ TRIUMF, 4004 Wesbrook Mall, Vancouver, BC, Canada, V6T 243
}

\begin{abstract}
Hadron masses are computed from quenched twisted mass lattice QCD for a degenerate doublet of up and down quarks with the twist angle set to $\pi / 2$, since this maximally twisted theory is expected to be free of linear discretization errors. Two separate definitions of the twist angle are used, and the hadron masses for these two cases are compared. The flavor breaking, that can arise due to twisting, is discussed in the context of mass splittings within the $\Delta(1232)$ multiplet.
\end{abstract}

\section{INTRODUCTION}

Twisted mass lattice quantum chromodynamics (tmLQCD) is an extension of the familiar Wilson lattice action 1, 2, 3]. TmLQCD is essentially obtained by introducing a chiralflavor rotation in the mass term of the Wilson action for a quark doublet (see Sec. III), and it offers a number of significant advantages. TmLQCD does not suffer from the "exceptional configurations" which arise in quenched and partially quenched Wilson simulations [4] due to the existence of unphysical zero modes. In tmLQCD, $O(a)$ improvement (where $a$ is the lattice spacing) can be implemented in a convenient manner. In particular at maximal twist $O(a)$ improvement is often automatic [5], that is, without the addition of a clover term [6]. The chiral nature of the rotation leads to parity mixings, but at maximal twist the mixings become mere operator interchanges. In addition, dynamical tmLQCD simulations [7, 8] are dramatically less expensive than dynamical chiral fermion simulations, and are claimed to be comparable to the cost of dynamical staggered fermions [9].

Nevertheless there are issues [7, 8, 10, 11] that remain to be understood. Of particular concern in this work is the definition of maximal twist. The original suggestion of Frezzotti and Rossi [5] was to obtain maximal twist by using the critical hopping parameter $\left(\kappa_{c W}\right)$ from the Wilson action. This procedure (which we refer to as the Wilson $\kappa_{c W}$ definition) was 
used in previously published computations [12, 13, 14]. However, recent theoretical studies [1, 15] have suggested that this is generally not precise enough to maintain tmLQCD's $O(a)$

improvement for small quark masses (for another point of view see Ref. [16]), and specific alternatives were presented.

In this work, the effect of using different definitions of maximal twist is studied by performing two separate simulations of the quenched hadron spectrum: one using the Wilson $\kappa_{c W}$ definition, and the other using a definition which arises by requiring that parity mixing, induced by the twisted mass term, vanish in a physical basis [8].

Our spectrum calculation includes pseudoscalar mesons, vector mesons, and baryons with spin-parity $(1 / 2)^{ \pm}$and $(3 / 2)^{ \pm}$for a degenerate $(\mathrm{u}, \mathrm{d})$ quark doublet. Computations were carried out at two lattice spacings and four quark masses.

Flavor breaking induced by the chiral-flavor rotation in tmLQCD should be suppressed by powers of the lattice spacing, but need not exactly vanish for a nonzero lattice spacing. Such an effect can be observed in principle as a mass splitting between charged and neutral pions, though the computation of a neutral pion mass involves diagrams with disconnected quark lines and is therefore expensive. Flavor breaking can also arise in a baryon multiplet, such as the isospin-3/2 $\Delta(1232)$, which contains states that are not related by $\mathrm{u}$ and $\mathrm{d}$ quark interchange. In this work, we calculate this mass splitting which does not involve any disconnected quark line contribution.

Section II contains a brief overview of tmLQCD and the determination of the maximal twist angle is discussed. Details of the simulations and the results are given in Sec. III. The discussion of flavor breaking is given separately is Sec. [V] and Sec. V] contains a summary.

\section{THE ACTION AT MAXIMAL TWIST}

The Lagrangian of continuum QCD for two massless quark flavors is invariant under the transformation

$$
\psi(x) \rightarrow e^{i \omega \gamma_{5} \tau^{3} / 2} \psi(x)
$$

where $\tau^{3}$ is the third Pauli matrix in flavor space and $\omega$ is called the twist angle. When a degenerate quark mass $m_{q}$ is added, the fermionic part of the Lagrangian is no longer 
invariant. After the transformation in Eq. (I) it becomes (in Euclidean notation)

$$
\mathcal{L}_{F}(x)=\bar{\psi}(x)\left(\not D+m+i \mu \gamma_{5} \tau^{3}\right) \psi(x),
$$

where $D_{\nu}$ is the covariant derivative and the parameters $m$ and $\mu$ will be called, respectively, the standard mass parameter and the twisted mass parameter. They are given by

$$
\begin{aligned}
m & =m_{q} \cos \omega, \\
\mu & =m_{q} \sin \omega .
\end{aligned}
$$

Two choices for the twist angle $\omega$ are of particular interest: $\omega=0$ causes $\mu$ to vanish and gives the QCD Lagrangian in its usual form, while $\omega=\pi / 2$ causes $m$ to vanish and is referred to as maximal twist. Note that for the continuum action the twist can not effect physics since it can be removed by a field redefinition.

One immediately sees that vector and axial currents get mixed by a general rotation. For example the charged currents,

$$
\begin{aligned}
V_{\nu}^{-}(x) & \equiv \bar{u}(x) \gamma_{\nu} d(x), \\
A_{\nu}^{-}(x) & \equiv \bar{u}(x) \gamma_{\nu} \gamma_{5} d(x),
\end{aligned}
$$

transform as follows:

$$
\begin{aligned}
V_{\nu}^{-}(x) & \rightarrow \tilde{V}_{\nu}^{-}(x) \cos \omega-i \tilde{A}_{\nu}^{-}(x) \sin \omega \\
A_{\nu}^{-}(x) & \rightarrow \tilde{A}_{\nu}^{-}(x) \cos \omega-i \tilde{V}_{\nu}^{-}(x) \sin \omega
\end{aligned}
$$

A tilde has been added to all currents constructed from fields in the twisted basis. Notice that the vector and axial currents don't mix at maximal twist, but their roles get interchanged.

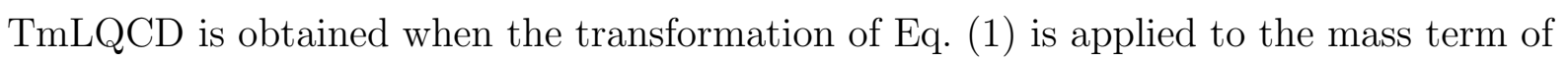
the Wilson lattice QCD action [2]. The fermion action used in the present work is in the twisted basis,

$$
S_{F}[\psi, \bar{\psi}, U]=a^{4} \sum_{x} \bar{\psi}(x)\left(M+i \mu \gamma_{5} \tau^{3}+\frac{1}{2} \sum_{\nu}\left(\gamma_{\nu} \nabla_{\nu}+\gamma_{\nu} \nabla_{\nu}^{*}-a \nabla_{\nu}^{*} \nabla_{\nu}\right)\right) \psi(x),
$$

where

$$
\begin{aligned}
& \nabla_{\nu} \psi(x)=U_{\nu}(x) \psi(x+a \hat{\nu})-\psi(x), \\
& \nabla_{\nu}^{*} \psi(x)=\psi(x)-U_{\nu}^{\dagger}(x-a \hat{\nu}) \psi(x-a \hat{\nu}) .
\end{aligned}
$$


In the lattice theory, both the mass term and the chiral symmetry breaking Wilson term lack invariance under Eq. (11) so, unlike the continuum case of Eq. (2), the chiral twist can not be removed from the action by a field redefinition. Essentially tmLQCD generalizes the Wilson fermion action by introducing a relative chiral phase between the mass term and the Wilson term.

The mass parameter $M$ in Eq. (9) undergoes both additive and multiplicative renormalization, but the twisted mass parameter $\mu$ is only renormalized multiplicatively. It is usual 2, 5] to divide $M$ into a mass shift $M_{s h}$ and a quark mass excess $m$ which plays the same role as the standard mass parameter in Eq. (2). In particular it is the quark mass excess which, along with $\mu$, determines the twist angle [2, 5]. Our quark propagator calculation will be done using the fermion action of Eq. (9) in the twisted basis so we dispense with this separation into mass shift and quark mass excess and simply quote $M$ as the standard mass parameter.

Various definitions of maximal twist are possible, and they will generally differ by lattice spacing effects. The Wilson $\kappa_{c W}$ definition of maximal twist [5] is obtained from the standard (unimproved) Wilson action $(\mu=0)$ by computing the pseudoscalar meson mass as a function of $M$ (equivalently the hopping parameter $\kappa$ ), then extrapolating to vanishing meson mass. This critical standard mass parameter $M_{c}$ is used for the mass shift $M_{s h}$ at all $\mu$ and simulations at $M=M_{c}, \mu \neq 0$ correspond to maximal twist in this definition. In terms of the hopping parameter, the critical standard mass parameter is

$$
a M_{c}=\frac{1}{2 \kappa_{c W}}-4
$$

This Wilson $\kappa_{c W}$ definition of maximal twist has been used previously in simulations 12, 13, 14], but studies of $O(a)$ improvement in twisted mass chiral perturbation theory recommend against it [11, 15]. Simulations with this definition are discussed in the present work as well, and are compared to a definition of maximal twist arising from considerations of parity conservation.

The tmLQCD action expressed in terms of twisted fields has a parity violating mass term. This term may be removed by a field redefinition and then the parity violation is associated with the Wilson term. The resulting form of the action is said to be in the physical basis 5]. The parity conservation definition of the twist angle comes from demanding the absence 
of a mixed pseudoscalar-vector matrix element in the physical basis [8, 15],

$$
\sum_{\vec{x}}\left\langle V_{\nu}^{-}(\vec{x}, t) P^{+}(0)\right\rangle=0,
$$

with

$$
P^{+}(x) \equiv \bar{d}(x) \gamma_{5} u(x) .
$$

Transforming this according to Eq. (7) and noting that $P^{+}(x)$ is invariant under the transformation of Eq. (11), we arrive at

$$
\tan \omega=\frac{i \sum_{\vec{x}}\left\langle\tilde{V}_{\nu}^{-}(\vec{x}, t) P^{+}(0)\right\rangle}{\sum_{\vec{x}}\left\langle\tilde{A}_{\nu}^{-}(\vec{x}, t) P^{+}(0)\right\rangle} .
$$

where a tilde again denotes currents constructed from fields in the twisted basis. This definition is essentially independent of Euclidean time $t$, as will be seen explicitly below. In this work, we compute the hadron spectrum for four values of $\mu$, with $M$ tuned in each case such that $\omega$ determined by Eq. (15) is $\pi / 2$.

\section{SIMULATIONS}

\section{A. Maximal twist from Wilson $\kappa_{c W}$}

In this section, the quenched hadron spectrum is obtained by using the Wilson $\kappa_{c W}$ definition of maximal twist. A standard heatbath algorithm with the Wilson plaquette action was used to create two quenched gauge field ensembles of 1000 configurations each, one at $\beta=5.85$ and the other at $\beta=6.0$, corresponding to lattice spacings near $a=0.123$ $\mathrm{fm}$ and $a=0.1 \mathrm{fm}$ respectively [12].

Twisted mass quark propagators are computed from the action in Eq. (91) with a periodic boundary condition in all directions using a 1-norm quasi-minimal residual algorithm. The standard mass parameter for each $\beta$ is set to the value obtained using $\kappa_{c W}$ from Ref. [12], and four different values for the twisted mass parameter are chosen which span the range of quark masses between $m_{s}$ and $m_{s} / 6$. ( $m_{s}$ denotes the strange quark mass.) From the point of view of numerical convergence of the propagator calculation, it would be possible to push the calculation to smaller quark masses. However, that would probably require even higher statistics to get meaningful results so for our first pass we have not tried to probe for the ultimate small quark mass limit. As it is, we achieve a pseudoscalar-meson to vector-meson 
mass ratio of about $1 / 3$. Numerical values of the simulation parameters are displayed in Table 【.

To uncover any finite volume corrections, simulations were run at $\beta=5.85$ on both $16^{3} \times 40$ and $20^{3} \times 40$ lattices. Differences in the mass spectrum were found to be negligible, so only results from the larger volume will be presented. Since the smaller volume was near $(2 \mathrm{fm})^{3}$, we chose a comparable physical volume for the $\beta=6.0$ simulations.

The ground state masses of the pion, $\rho$ meson, $(1 / 2)^{ \pm}$baryons and $(3 / 2)^{ \pm}$baryons are obtained from the following operators, defined in the physical quark basis:

$$
\begin{aligned}
\pi^{ \pm} & =\bar{\psi} \gamma_{5} \tau^{\mp} \psi \\
\rho_{i}^{ \pm} & =\bar{\psi} \gamma_{i} \tau^{\mp} \psi \\
\tilde{\rho}_{i}^{ \pm} & =\bar{\psi} \sigma_{i 4} \tau^{\mp} \psi \\
p & =\epsilon_{a b c}\left[u_{a}^{T} C \gamma_{5} d_{b}\right] u_{c} \\
n & =\epsilon_{a b c}\left[d_{a}^{T} C \gamma_{5} u_{b}\right] d_{c} \\
\Delta_{i}^{++} & =\epsilon_{a b c}\left[u_{a}^{T} C \gamma_{i} u_{b}\right] u_{c} \\
\Delta_{i}^{+} & =\frac{2}{\sqrt{3}} \epsilon_{a b c}\left[u_{a}^{T} C \gamma_{i} d_{b}\right] u_{c}+\frac{1}{\sqrt{3}} \epsilon_{a b c}\left[u_{a}^{T} C \gamma_{i} u_{b}\right] d_{c}, \\
\Delta_{i}^{0} & =\frac{2}{\sqrt{3}} \epsilon_{a b c}\left[d_{a}^{T} C \gamma_{i} u_{b}\right] d_{c}+\frac{1}{\sqrt{3}} \epsilon_{a b c}\left[d_{a}^{T} C \gamma_{i} d_{b}\right] u_{c}, \\
\Delta_{i}^{-} & =\epsilon_{a b c}\left[d_{a}^{T} C \gamma_{i} d_{b}\right] d_{c},
\end{aligned}
$$

where $C$ is the charge conjugation matrix, $i$ is a spatial Lorentz index, and sums over color indices $a, b$ and $c$ are implied. Since the $\rho_{i}$ operator tends to produce a somewhat noisy correlator in tmLQCD simulations, the operator $\tilde{\rho}_{i}$ is also considered.

As is well known, the $\Delta_{i}$ operators contain a spin $1 / 2$ admixture. At zero momentum, the correlator for spatial Lorentz indices is 17 ]

$$
\sum_{\vec{x}}\left\langle 0\left|T\left(\Delta_{j}(\vec{x}, t) \bar{\Delta}_{i}(\overrightarrow{0}, 0)\right)\right| 0\right\rangle \equiv G_{j i}(t)=\left(\delta_{j i}-\frac{1}{3} \gamma_{j} \gamma_{i}\right) G_{3 / 2}(t)+\frac{1}{3} \gamma_{j} \gamma_{i} G_{1 / 2}(t) .
$$

The spins are easily separated,

$$
\begin{aligned}
G_{1 / 2}(t) & =\frac{1}{3} \operatorname{Tr}\left[G_{j i}(t) \gamma_{i} \gamma_{j}\right] \\
G_{3 / 2}(t) & =\frac{1}{6} \operatorname{Tr}\left[G_{j i}(t) \gamma_{j} \gamma_{i}+C_{i i}(t)\right],
\end{aligned}
$$

where repeated indices are summed. We will use this $G_{3 / 2}(t)$ to determine the masses of spin $3 / 2$ baryons. 
To get twisted versions of the operators in Eqs. (16]24), one simply applies the twist from Eq. (11). In practice the simulation is done by computing the tmLQCD quark propagators in the twisted basis using Eq. (91) and then applying Eq. (11) to both ends. The correlators then involve the operators unchanged in the physical basis.

As discussed in Ref. [18], baryon two-point correlators couple to both parities even without twisting. For periodic boundary conditions, the correlator is

$$
G(t)=\sum_{s= \pm}\left[\left(1+s \gamma_{4}\right) e^{-M_{s} t}+\left(1-s \gamma_{4}\right) e^{-M_{s}(T-t)}\right] s G^{(s)}(t),
$$

where $T$ is the temporal extent of the lattice, and $s$ sums over positive and negative parity. This expression is directly applicable to our tmLQCD simulations at maximal twist as well. We can therefore discuss the ground state masses of both parities from the baryon operators in Eqs. (19]|24).

Throughout this work, all hadron masses are obtained from three-exponential fits to the hadron correlators using all time slices except the source. Statistical uncertainties are obtained from the bootstrap method with replacement, where the number of bootstrap ensembles is three times the number of data points in the original ensemble.

Our pion mass squared is obtained from the configuration average of $\pi^{+}$and $\pi^{-}$and is plotted versus $\mu$ in Fig. 1. Excellent agreement is obtained with the $\beta=5.85$ tmLQCD results from Ref. [13]. A least squares fit of our data to the form

$$
\left(a m_{\pi}\right)^{2}=A+B(a \mu)+C(a \mu)^{2}
$$

is also displayed. Notice that the fitted curve does not lead to a vanishing pion mass for $\mu=0$, an effect also noted in Ref. [13]. There could be various reasons for this but one's first guess might be that it is due to lattice spacing errors in the determination of $\kappa_{c W}$ and of the twist angle. We will revisit this issue, and also consider the pion decay constant, in Sec. IIIB in the context of an alternate definition of maximal twist.

Fig. 2 is a plot of vector meson mass, averaged over $\rho^{+}$and $\rho^{-}$and over spatial Lorentz indices, versus pseudoscalar mass squared, again with tmLQCD results from Ref. 13] for comparison. It is interesting to see that the operator from Eq. (18) has consistently smaller uncertainties than the traditional vector operator of Eq. (17). It is also noted that a linear extrapolation to vanishing pion mass squared produces a vector mass that agrees with the experimental $\rho$ meson mass, at both $\beta$ values. 
Figures 3 and 4 show the baryon masses obtained from our fits along with positive parity results from Ref. 13] for comparison. In these plots, our nucleon is the configuration average of proton and neutron, while our $\Delta$ is the configuration average of $\sum_{i=1}^{3} \Delta_{i}^{++}$and $\sum_{i=1}^{3} \Delta_{i}^{-}$. Extrapolations of the positive parity baryons are within about $10 \%$ of the experimental masses. The connection of negative parity data to experiment is less clear.

The $O(a)$ improvement of tmLQCD should result in better scaling between lattices at $\beta=5.85$ and $\beta=6.0$ than would be obtained from the Wilson action. This was studied for the mass spectrum in Ref. [12] and discussed for the pion form factor in Ref. [14]. Our present data for the $\rho$ and nucleon masses have only a very small dependence on lattice spacing relative to the statistical uncertainties, as displayed in Figure 5 .

\section{B. Maximal twist from parity conservation}

The Wilson definition of maximal twist used in Sec. IIA relies on Eq. (12) with the numerical values of $\kappa_{c W}$ taken from Ref. [12]. In this section, maximal twist will be defined by tuning using Eq. (15). To show that the two options are numerically quite different, Fig. 6 displays $\omega$ as obtained from Eq. (15) when the standard and twisted mass parameters keep the values used in Sec. IIIA. The source is at (Euclidean) time 1 in this plot. Each $\mu$ value is found to correspond to a different twist angle $\omega$, and the angles range from $65^{\circ}$ to more than $90^{\circ}$.

In light of cautionary notes against relying on the Wilson $\kappa_{c W}$ definition of maximal twist [11, 15], we will now tune $\omega$ to $\pi / 2$ according to Eq. (15) and recompute the mass spectrum. Parameters used in the simulations are listed in Table 凹. Notice in particular that these results use 300 gauge field configurations as opposed to the 1000 configurations used for the $\kappa_{c W}$ definition of maximal twist in Sec. IIIA.

Fig. 7 is a plot of the standard mass parameter $M$ tuned such that $\omega=\pi / 2$ for each twisted mass parameter. The dependence of $a M$ on $a \mu$ is seen to be quite linear with a distinctly nonzero slope.

The pseudoscalar meson mass squared is plotted for both definitions of maximal twist in Fig. 8. The least squares fit of each to a quadratic is also shown. It is evident that the parity conservation definition of maximal twist, i.e. Eq. (15), produces a smaller pion mass for sufficiently small $\mu$, thus demonstrating that the $\kappa_{c W}$ definition did not precisely correspond 
to $\omega=\pi / 2$ for this observable. In addition, Fig. 8 indicates that the parity conservation definition of maximal twist leads to less curvature in the $m_{\pi}^{2}$ versus $\mu$ extrapolation and a smaller intercept for $m_{\pi}^{2}$ at $\mu=0$.

The curvature of $m_{\pi}^{2}$ as a function of quark mass and the non-zero value at zero quark mass are features commonly found in lattice simulations. Higher order lattice spacing errors may lead to a residual pion mass even after tuning to maximal twist to remove $O(a)$ effects. Finite volume effects can not be discounted, although our simulation at $\beta=5.85$ in Sec. IIA did not give any indication of such a problem. In addition, the simple extrapolation function Eq. (29), although consistent with simulation data, may not be appropriate at very small quark masses. Quenched chiral perturbation theory suggests a non-analytic behavior of $m_{\pi}^{2}$ at zero quark mass $[19,20,21]$.

The chiral fits of Chen et al. 22] to a large set of pion masses calculated at very small quark masses (using the overlap fermion approach) are very instructive. They show how quenched chiral logarithms can conspire to fit simulation data with an essentially linear quark mass behavior in $m_{\pi}^{2}$ with the pion mass dipping down to zero only at extremely small quark masses. That paper also contains many references to earlier searches for quenched chiral logarithms.

It may be interesting to look for quenched chiral logarithms with tmLQCD. However Ref. 22] suggests much more data at quark masses smaller than those considered in this work would be needed. If one has to tune the standard mass parameter as a function of the twisted mass $\mu$, this would add to the overall cost of such a calculation.

In addition to the pion mass, our simulations provide a determination of the pion decay constant. The "indirect" method of Ref. [13] is particularly convenient,

$$
f_{\pi}=\frac{2 \mu}{m_{\pi}^{2}}\left|\left\langle 0\left|P^{ \pm}\right| \pi\right\rangle\right|,
$$

where the normalization is such that the physical value is $f_{\pi} \approx 130 \mathrm{MeV}$. Fig. 9 compares the results for the two definitions of maximal twist. Numerical results from Ref. [13] using the Wilson $\kappa_{c W}$ definition are also displayed. Much like the pion mass squared, the decay constant has significantly less curvature in the quark mass when the parity conservation definition of maximal twist is used, and is consistent with a linear function of $m_{\pi}^{2}$ as expected from chiral perturbation theory. In contrast, data from the Wilson $\kappa_{c W}$ definition are clearly not linear and do not approach a realistic value. Fig. 10 shows that the decay constant 
is independent of lattice spacing for all quark masses within uncertainties for the parity definition, while the $\kappa_{c W}$ definition does not scale as well.

Beyond the pseudoscalar meson, there is very little difference in the computed spectrum between the two definitions of maximal twist. The $\rho$ meson, spin- $1 / 2$ baryons and spin- $3 / 2$ baryons are displayed in Figs. 11, 12 and 13 respectively. Clear signals are obtained for the $\rho$, nucleon and $\Delta$ hadrons, but data for the negative parity states do not show a convincing signal. The $\rho$ meson and nucleon masses can be seen to be independent of lattice spacing in Fig. 14 over the whole range of quark masses that were investigated.

\section{FLAVOR BREAKING AND THE $\Delta(1232)$ MULTIPLET}

For the Wilson lattice action $(\mu=0)$ with degenerate quark masses, the up and down quark propagators are identical so hadron masses within any isospin multiplet are degenerate. In tmLQCD, the up and down propagators (let's name them $U(x, y)$ and $D(x, y)$ ) differ by having opposite signs for the $\mu$ term, so conservation of isospin is not guaranteed. However, tmLQCD does satisfy

$$
\begin{aligned}
& U(x, y)=\gamma_{5} D^{\dagger}(y, x) \gamma_{5} \\
& D(x, y)=\gamma_{5} U^{\dagger}(y, x) \gamma_{5}
\end{aligned}
$$

This leads to the equality of any given hadron two-point correlator with its up-down interchanged hermitian conjugate. Since masses are obtained from the real parts of correlators, we arrive at the following degeneracies:

$$
\begin{gathered}
\pi^{+} \text {and } \pi^{-}, \\
\rho^{+} \text {and } \rho^{-}, \\
\text {proton and neutron, } \\
\Delta^{++} \text {and } \Delta^{-}, \\
\Delta^{+} \text {and } \Delta^{0}
\end{gathered}
$$

The difference between charged and neutral pion masses offers a measure of isospin violation, though it would require the computation of disconnected quark diagrams. Here, we consider a less expensive alternative: $m_{\Delta^{++,-}}-m_{\Delta^{+, 0}}$.

Figs. 15] and 16] show our results for this $\Delta$ splitting in physical units, as obtained respectively from Wilson $\kappa_{c W}$ maximal twist and parity maximal twist. No significant difference 
is found between the two definitions of maximal twist for this observable. Based on the better statistics of Fig. 15] (1000 configurations rather than only 300), we see evidence that $m_{\Delta^{++,-}}-m_{\Delta^{+, 0}}$ decreases with decreasing lattice spacing, as expected.

\section{SUMMARY}

Twisted mass lattice QCD offers significant advantages over the usual Wilson or clover [6] actions. First "exceptional configurations" are eliminated and secondly there is the possibility, by tuning the mass parameter, of removing $O(a)$ lattice spacing errors automatically. Our results add to the evidence that these advantages are realized in numerical simulations. The spectrum of hadron masses was computed in tmLQCD for two lattice spacings and two definitions of maximal twist (where the spectrum is expected to be automatically $O(a)$ improved). Four values of the quark mass were used, the lightest corresponding to a pseudoscalar-meson to vector-meson mass ratio of about $1 / 3$. No problems with convergence of the quark propagator calculation were observed so the quark mass could be pushed even lower.

There are different ways to define the twist angle and hence determine maximal twist. With the parity conservation definition of maximal twist, Eq. (15), the pion decay constant was found to be independent of lattice spacing for all quark masses, though this was not true for the definition of maximal twist that relies on the Wilson critical hopping parameter. No significant lattice spacing dependence in vector meson or nucleon masses was observed over the whole range of quark mass for both definitions of maximal twist. Additional calculations at more lattice spacings are needed before definitive statements about scaling can be made. However, it is already clear that maximally-twisted tmLQCD is competitive with other improved actions and, of course, much better than the unimproved Wilson action.

Phenomenologically reasonable results were obtained for the pion, $\rho$ meson, nucleon and $\Delta(1232)$. The tensor operator was found to be useful for attaining the $\rho$ mass with a smaller uncertainty. Hints of the negative parity baryons were also obtained. To do better with the baryon resonances probably requires a more sophisticated treatment with operators optimized for particular states [23].

For a fixed value of the twisted mass parameter $\mu$, the parity conservation definition of maximal twist produced a smaller pion mass than the Wilson $\kappa_{c W}$ definition. Since the 
pion mass squared should be minimized at maximal twist, this suggests that the parity conservation definition is a better definition, at least for this observable. In addition, only the parity conservation definition led to a realistic extrapolation for the pion decay constant. Note that implementing this definition required tuning of the standard mass parameter for every twisted mass value which adds to the overall cost of doing the calculations. For the rest of the mass spectrum, our data do not show a statistically significant difference between the two definitions of maximal twist.

A lattice artifact of tmLQCD is flavor symmetry breaking. This can manifest itself as a mass difference between charged and neutral pions. However, the calculation of the neutral pion mass in the presence of flavor symmetry breaking is a formidable task since it involves disconnected quark line contributions. In this work we considered mass splittings in the isospin-3/2 $\Delta(1232)$ multiplet which involved no disconnected quark line contribution. From our higher statistics run there is evidence that the flavor symmetry breaking effect is present and that it decreases at smaller lattice spacing.

\section{Acknowledgments}

This work was supported in part by the Natural Sciences and Engineering Research Council of Canada, the Canada Foundation for Innovation, the Canada Research Chairs Program and the Government of Saskatchewan.

[1] R. Frezzotti, P. A. Grassi, S. Sint and P. Weisz Nucl. Phys. B, Proc. Suppl. 83, 941 (2001).

[2] R. Frezzotti, P. A. Grassi, S. Sint and P. Weisz [Alpha Collaboration], J. High Energy Phys. 08, 058 (2001).

[3] ALPHA Collaboration (M. Della Morte et al.), J. High Energy Phys. 10, 041 (2001); C. McNeile and C. Michael, Nucl. Phys. B, Proc. Suppl. 106, 251 (2002).

[4] W. Bardeen, A. Duncan, E. Eichten, G. Hockney, and H. Thacker, Phys. Rev. D 57, 1633 (1998).

[5] R. Frezzotti and G. C. Rossi, J. High Energy Phys. 08, 007 (2004).

[6] B. Sheikholeslami and R. Wohlert, Nucl. Phys. B259, 572 (1985). 
[7] F. Farchioni et al.., Eur. Phys. J. C39, 421 (2005).

[8] F. Farchioni et al., hep-lat/0410031.

[9] A. D. Kennedy, hep-lat/0409167

[10] S. R. Sharpe and J. M. S. Wu, Phys. Rev. D 70, 094029 (2004).

[11] S. Aoki and O. Bär, Phys. Rev. D 70, 116011 (2004).

[12] K. Jansen, A. Shindler, C. Urbach and I. Wetzorke, Phys. Lett. B 586, 432 (2004).

[13] $\chi$ LF Collaboration (W. Bietenholz et al.), J. High Energy Phys. 12, 044 (2004).

[14] A. M. Abdel-Rehim and R. Lewis, Phys. Rev. D 71, 014503 (2005).

[15] S. R. Sharpe and J. M. S. Wu, hep-lat/0411021

[16] R. Frezzotti, hep-lat/0409138.

[17] M. Benmerrouche, R. M. Davidson and N. C. Mukhopadhyay, Phys. Rev. C 39, 2339 (1989).

[18] S. Sasaki, T. Blum and S. Ohta, Phys. Rev. D 65, 074503 (2002).

[19] C. W. Bernard and M. F. L. Golterman, Phys. Rev. D 46, 853 (1992).

[20] S. R. Sharpe, Phys. Rev. D 46, 3146 (1992).

[21] G. Münster, C. Schmidt and E. E. Scholz, Europhys. Lett. 86, 639 (2004).

[22] Y. Chen et al., Phys. Rev. D 70, 034502 (2004).

[23] LHP Collaboration (S. Basak et al.), Nucl. Phys. B, Proc. Suppl. 128, 186 (2004). 
TABLE I: Parameters used in the simulations. Parameters in parenthesis denote the one instance where two definitions of maximal twist happen to coincide so new data were not generated.

\begin{tabular}{|c|c|c|c|c|}
\hline$\beta$ & \#sites & \#configurations & $a M$ & $a \mu$ \\
\hline \multirow[t]{8}{*}{5.85} & $20^{3} \times 40$ & 1000 & -0.9071 & 0.0376 \\
\hline & & & -0.9071 & 0.0188 \\
\hline & & & -0.9071 & 0.01252 \\
\hline & & & -0.9071 & 0.00627 \\
\hline & & 300 & -0.8965 & 0.0376 \\
\hline & & & $(-0.9071)$ & $(0.0188)$ \\
\hline & & & -0.9110 & 0.01252 \\
\hline & & & -0.9150 & 0.00627 \\
\hline \multirow[t]{8}{*}{6.0} & $20^{3} \times 48$ & 1000 & -0.8135 & 0.030 \\
\hline & & & -0.8135 & 0.015 \\
\hline & & & -0.8135 & 0.010 \\
\hline & & & -0.8135 & 0.005 \\
\hline & & 300 & -0.8110 & 0.030 \\
\hline & & & -0.8170 & 0.015 \\
\hline & & & -0.8195 & 0.010 \\
\hline & & & -0.8210 & 0.005 \\
\hline
\end{tabular}


TABLE II: The mass spectrum and pion decay constant from our simulations. The vector meson masses are obtained from $\bar{\psi} \sigma_{j 4} \tau^{ \pm} \psi$ or $\bar{\psi} \gamma_{j} \tau^{ \pm} \psi$, whichever has the smaller uncertainty. Spin $3 / 2$ masses are given as averages over pairs within the multiplet.

\begin{tabular}{|c|c|c|c|c|c|c|c|c|}
\hline$\beta$ & $a M$ & $a \mu$ & $a m_{\pi}$ & $a f_{\pi}$ & $a m_{\rho}$ & $a m_{N}$ & $a m_{\Delta^{++}, \Delta^{-}}$ & $a m_{\Delta^{+}, \Delta^{0}}$ \\
\hline \multirow[t]{7}{*}{5.85} & -0. & 76 & $0.4341(4)$ & 0.114 & $0.647(4)$ & 0.94 & $1.079(6)$ & $1.056(6)$ \\
\hline & -0.9071 & 0.0188 & ) & 0.09 & $566(8)$ & ) & $.976(9)$ & $0.933(11)$ \\
\hline & -0.9071 & 0.01252 & 5) & 7) & 0 . & 0.7 & 1) & 6) \\
\hline & -0.9071 & 0.00627 & $0.1837(6)$ & 0.08 & 0.49 & $0.682(11)$ & $0.884(15)$ & $0.863(22)$ \\
\hline & -0.8965 & 0.0376 & 0.42 & 0.1 & 0.6 & 0.92 & 5) & 0) \\
\hline & -0.9110 & 0.01 & $0.2509(8)$ & $0.0933(10)$ & $0.559(15)$ & $0.751(13)$ & $0.93^{\prime}$ & $0.851(30)$ \\
\hline & -0.91 & 0.00627 & 0.17 & $0.0876(11)$ & $4(3)$ & $5(22)$ & $0.886(33)$ & 0.81 \\
\hline \multirow[t]{8}{*}{6.0} & -0.8135 & 0.030 & $0.3332(5)$ & 0.08 & 0.4 & 0.7 & (4) & $0.830(5)$ \\
\hline & -0.81 & 0.015 & 0.2 & $0.0^{\prime}$ & 0. & 0.6 & (7) & $0.760(8)$ \\
\hline & -0.8135 & 0.010 & 0.197 & 0.06 & 0.4 & 0.5 & (8) & 0.73 \\
\hline & -0.81 & $0 . C$ & 0.1 & 0.0 & (11) & 0.5 & 0.7 & 0.7 \\
\hline & -0.8110 & 0.030 & $0.3320(9)$ & 0.08 & $0.482(5)$ & $0.719(5)$ & $0.839(7)$ & $0.821(7)$ \\
\hline & -0.8170 & 0.015 & 0.23 & 0.075 & $0.424(12)$ & $0.613(10)$ & $0.786(13)$ & $0.753(14)$ \\
\hline & -0.8195 & 0.010 & $0.1944(12)$ & $0.0723(10)$ & $0.417(20)$ & $0.571(14)$ & $0.772(18)$ & $0.724(20)$ \\
\hline & -0.8210 & 0.005 & $0.1404(17)$ & $0.0673(11)$ & $0.412(50)$ & $0.514(20)$ & $0.758(27)$ & $0.688(37)$ \\
\hline
\end{tabular}



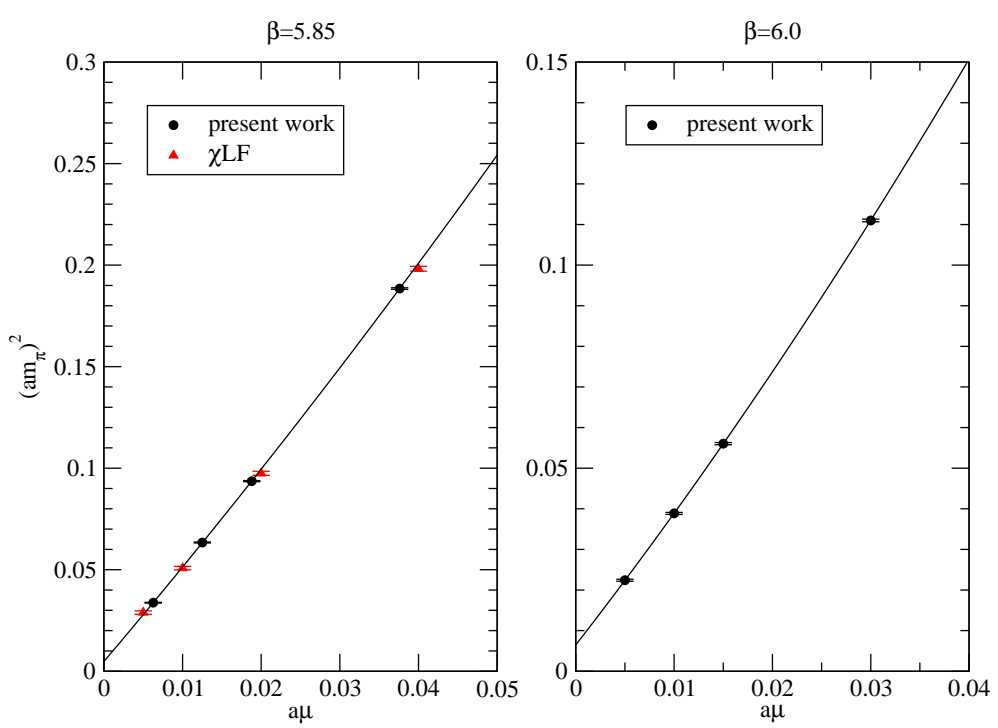

FIG. 1: Pseudoscalar meson mass squared as a function of the twisted mass parameter. The standard mass parameter was held fixed to the value obtained from the Wilson $\kappa_{c W}$ definition of maximal twist, Eq. (12). Data labelled by " $\chi$ LF" are taken from Ref. [13].
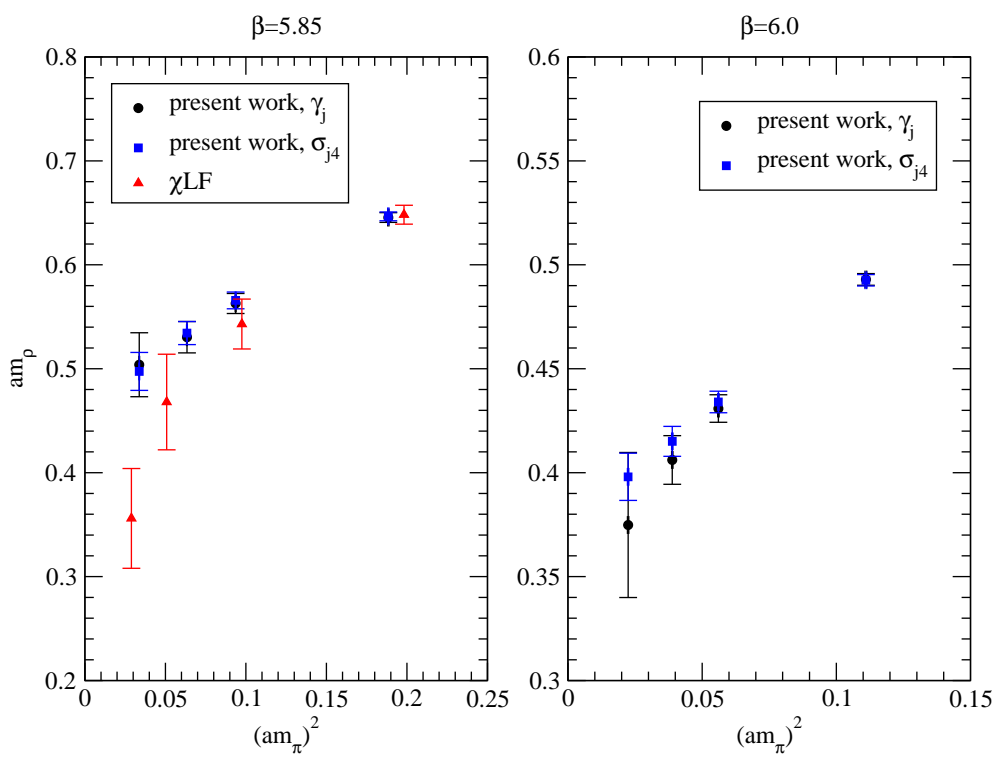

FIG. 2: Vector meson mass as a function of the pseudoscalar meson mass squared. The standard mass parameter was held fixed to the value obtained from the Wilson $\kappa_{c W}$ definition of maximal twist, Eq. (12). Data labelled by “ $\chi$ LF" are taken from Ref. [13]. 

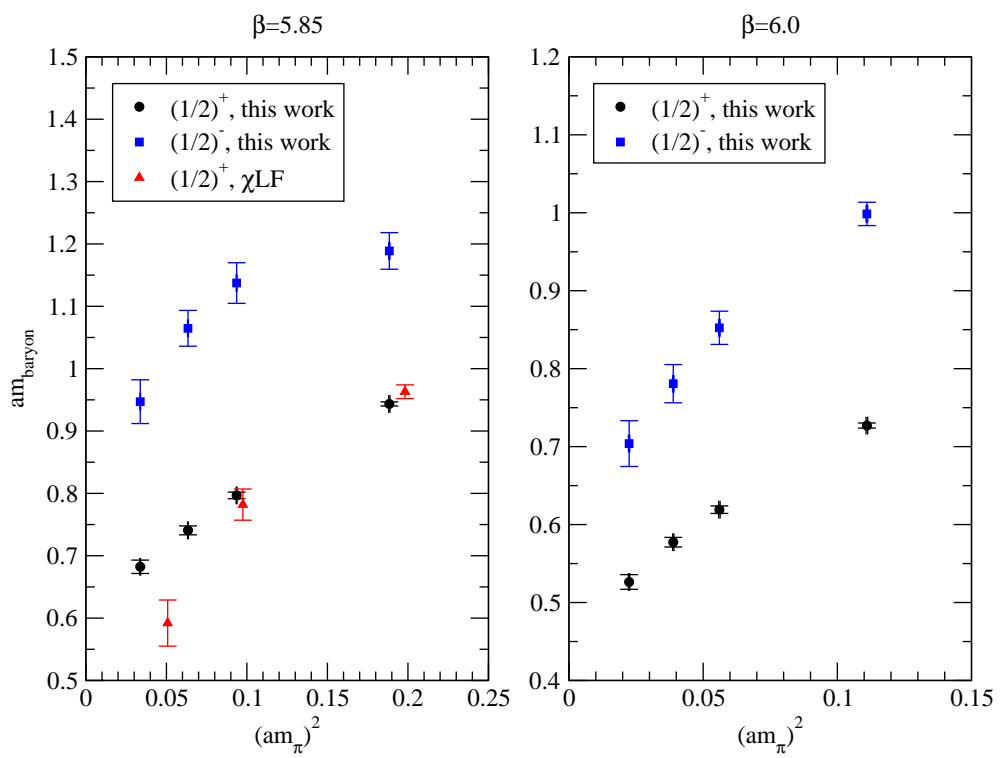

FIG. 3: Spin 1/2 baryon masses (both parities) as functions of the pseudoscalar meson mass squared. The standard mass parameter was held fixed to the value obtained from the Wilson $\kappa_{c W}$ definition of maximal twist, Eq. (12). Data labelled by " $\chi$ LF" are taken from Ref. 13].
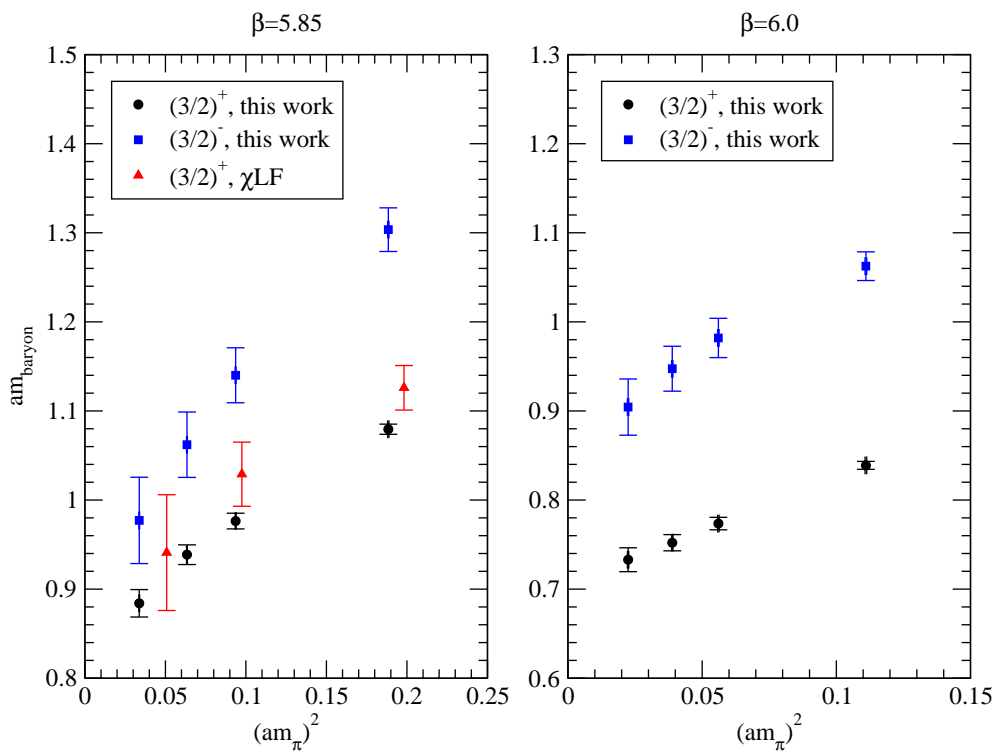

FIG. 4: Spin 3/2 baryon masses (both parities) as functions of the pseudoscalar meson mass squared. These data are averages of the $\Delta^{++}$and $\Delta^{-}$masses. The standard mass parameter was held fixed to the value obtained from the Wilson $\kappa_{c W}$ definition of maximal twist, Eq. (12). Data labelled by " $\chi \mathrm{LF} "$ are taken from Ref. [13]. 

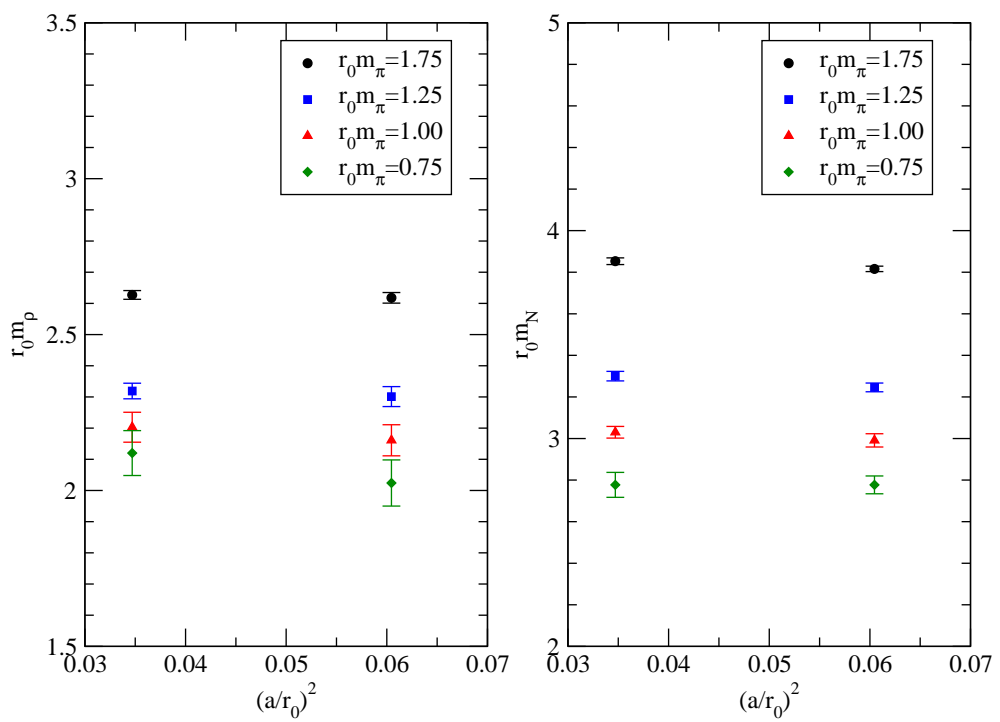

FIG. 5: Vector meson and $(1 / 2)^{+}$baryon masses as functions of lattice spacing squared. Axes are in units of $r_{0}=0.5 \mathrm{fm}$. The calculation was done using the Wilson $\kappa_{c W}$ definition of maximal twist, Eq. (12).
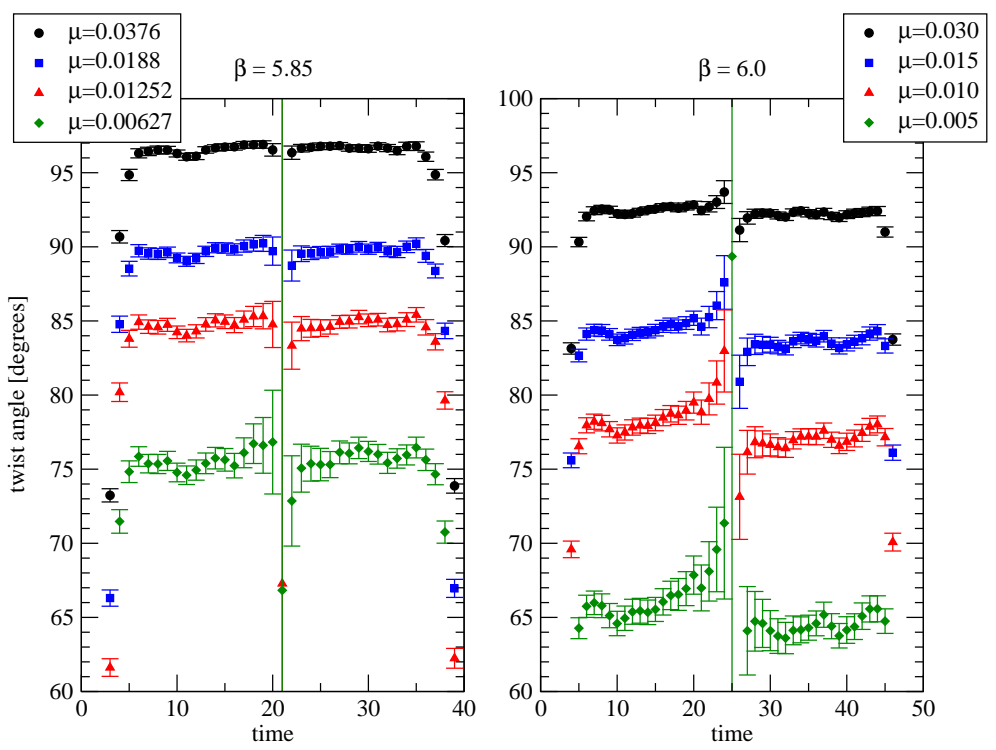

FIG. 6: The twist angle obtained from Eq. (15) at different lattice time slices while using the Wilson $\kappa_{c W}$ definition of maximal twist, Eq. (12). 


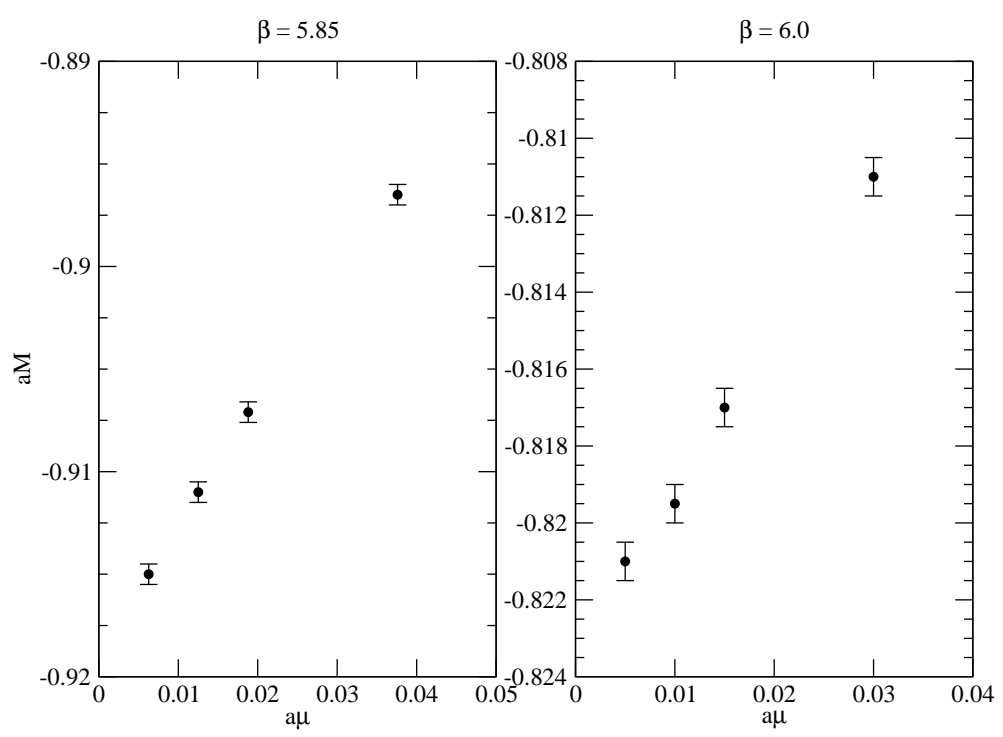

FIG. 7: The standard mass parameter as a function of the twisted mass parameter, tuned such that the twist angle $\omega$ of Eq. (15) is $\pi / 2$.
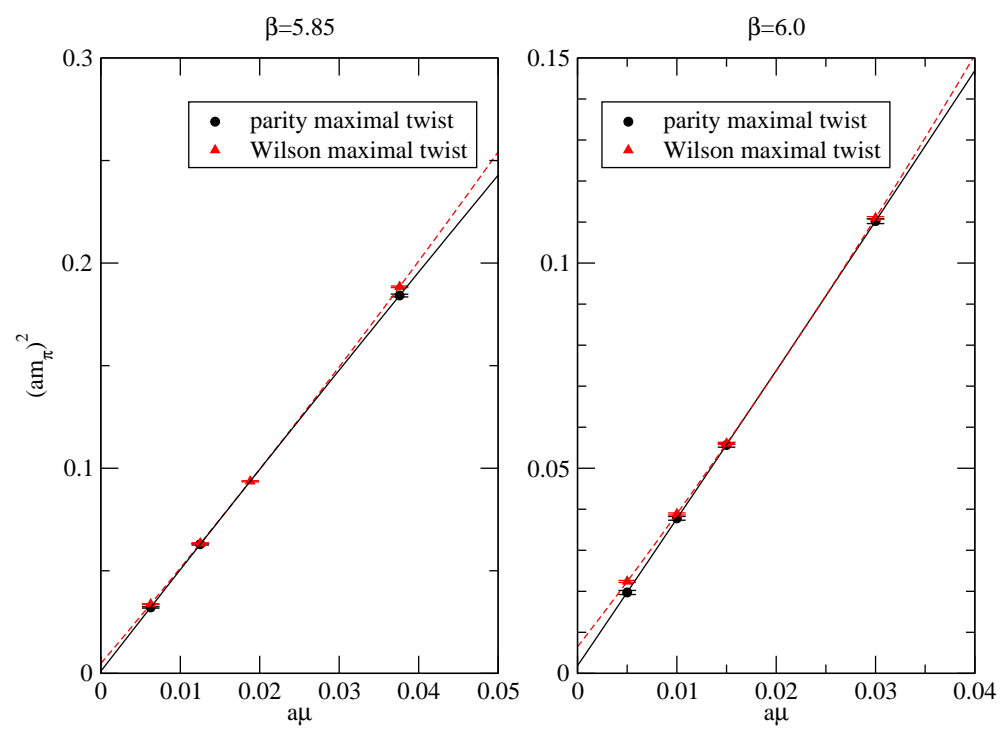

FIG. 8: Pseudoscalar meson mass squared as a function of the twisted mass parameter, calculated using the parity conservation definition of maximal twist: $\omega=\pi / 2$ in Eq. (15). Data calculated using the Wilson $\kappa_{c W}$ definition, Fig. 1. are shown for comparison. 

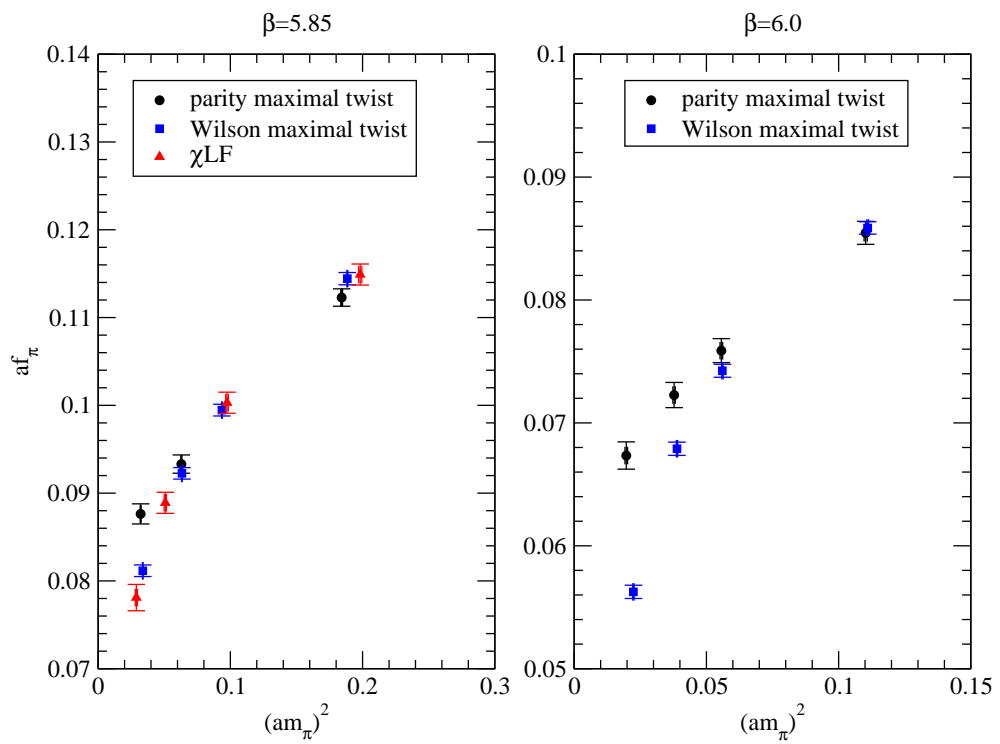

FIG. 9: Pseudoscalar meson decay constant as a function of the pseudoscalar meson mass squared, calculated using both the parity conservation definition and the Wilson $\kappa_{c W}$ definition of maximal twist. Data labelled by " $\chi$ LF" are taken from Ref. [13].
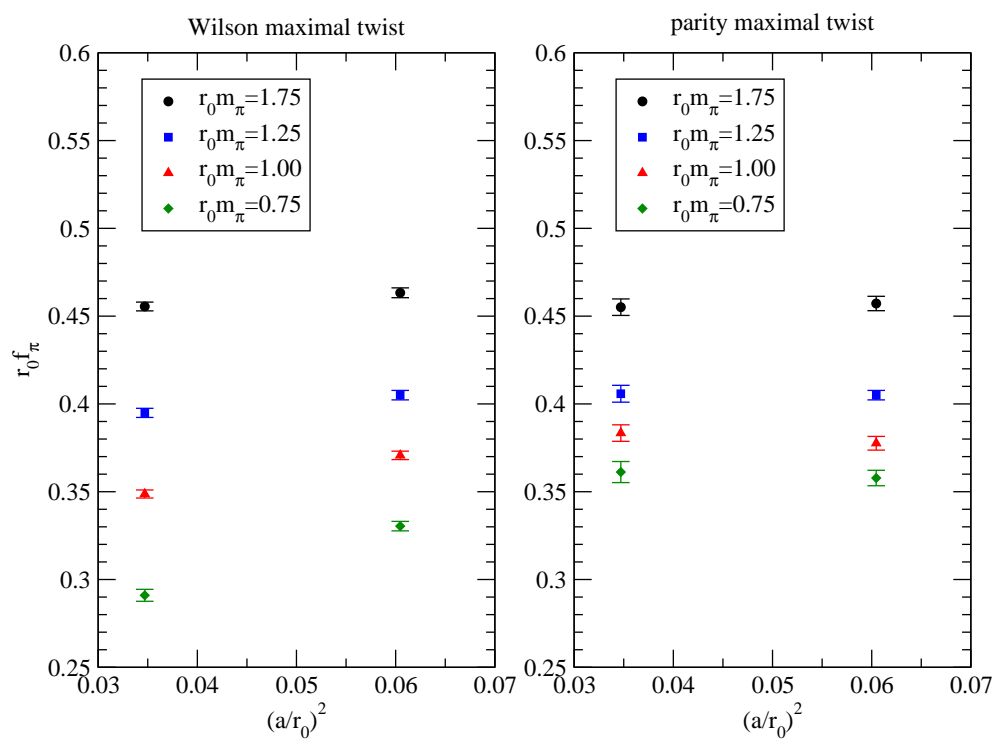

FIG. 10: Pseudoscalar meson decay constant as a function of lattice spacing squared, calculated using both the parity conservation definition and the Wilson $\kappa_{c W}$ definition of maximal twist. Axes are in units of $r_{0}=0.5 \mathrm{fm}$. 

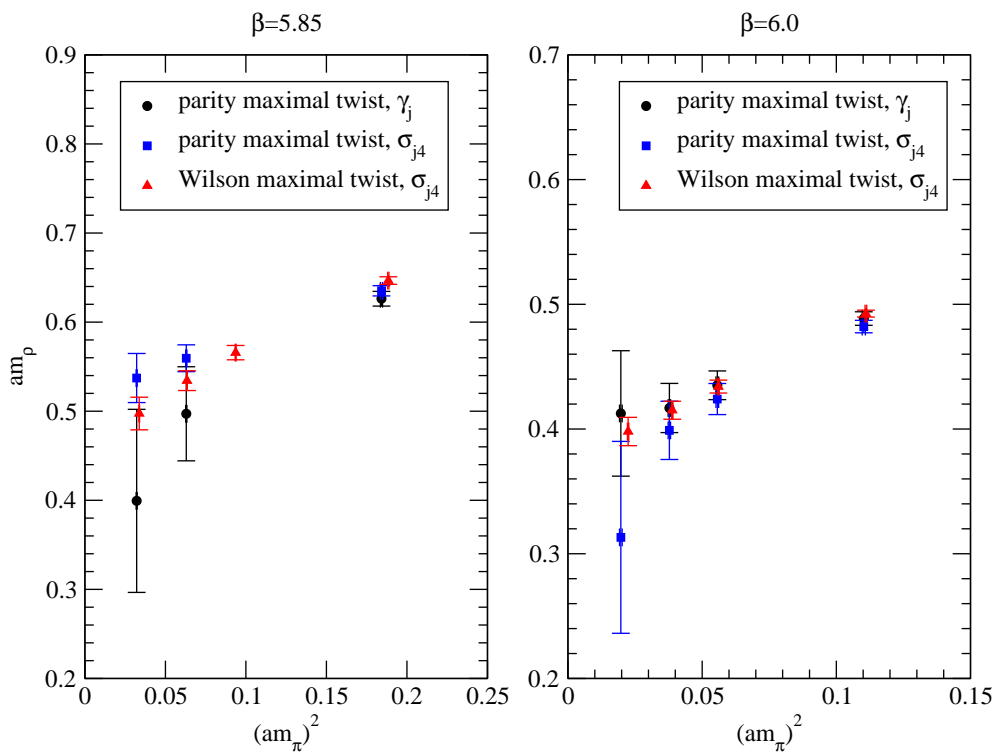

FIG. 11: Vector meson mass as a function of the pseudoscalar meson mass squared, calculated using the parity conservation definition of maximal twist: $\omega=\pi / 2$ in Eq. (15). Data calculated using the Wilson $\kappa_{c W}$ definition, Fig. 1] are shown for comparison.
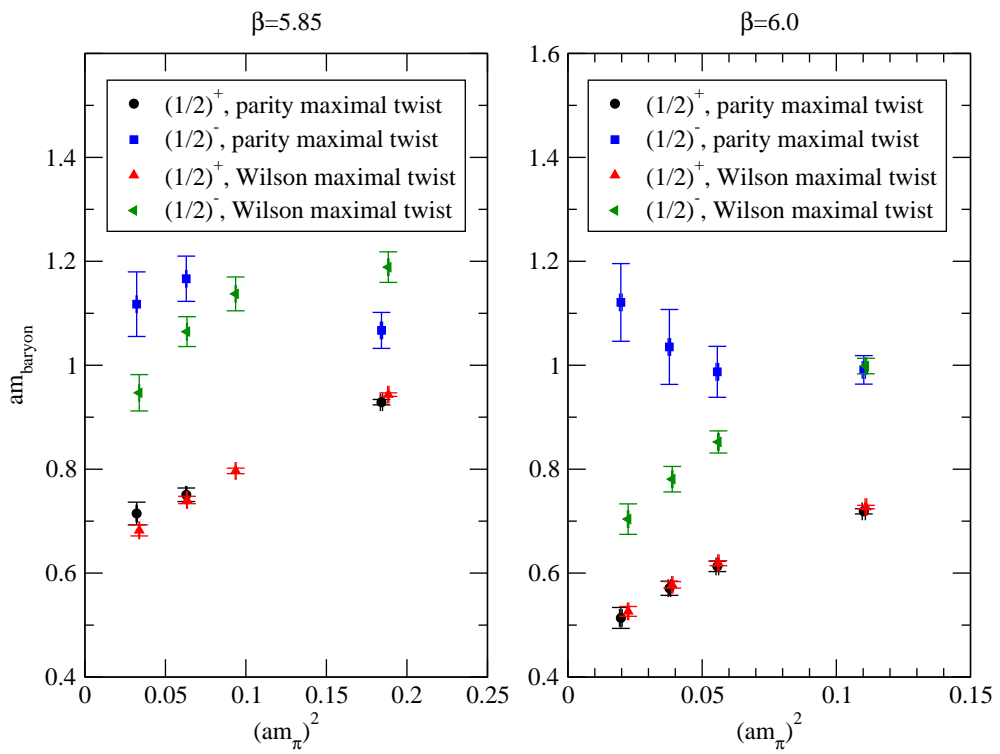

FIG. 12: Spin 1/2 baryon masses (both parities) as functions of the pseudoscalar meson mass squared, calculated using the parity conservation definition of maximal twist: $\omega=\pi / 2$ in Eq. (15). Data calculated using the Wilson $\kappa_{c W}$ definition, Fig. 1 are shown for comparison. 

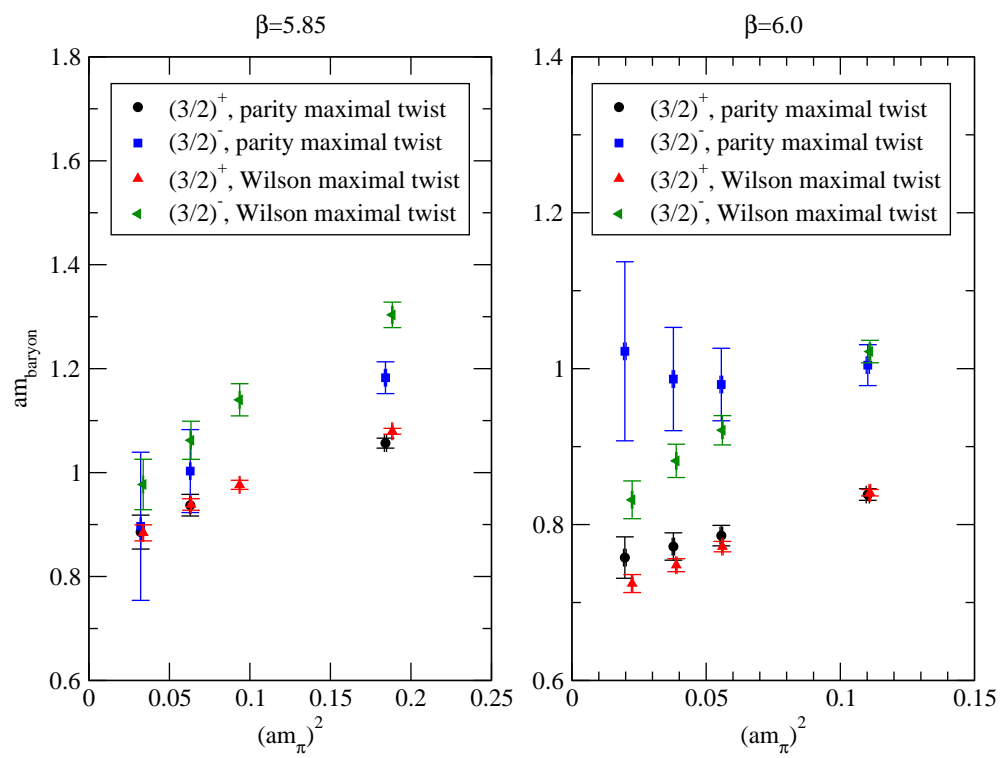

FIG. 13: Spin 3/2 baryon masses (both parities) as functions of the pseudoscalar meson mass squared, calculated using the parity conservation definition of maximal twist: $\omega=\pi / 2$ in Eq. (15). These data are averages of the $\Delta^{++}$and $\Delta^{-}$masses. Data calculated using the Wilson $\kappa_{c W}$ definition, Fig. 1] are shown for comparison.
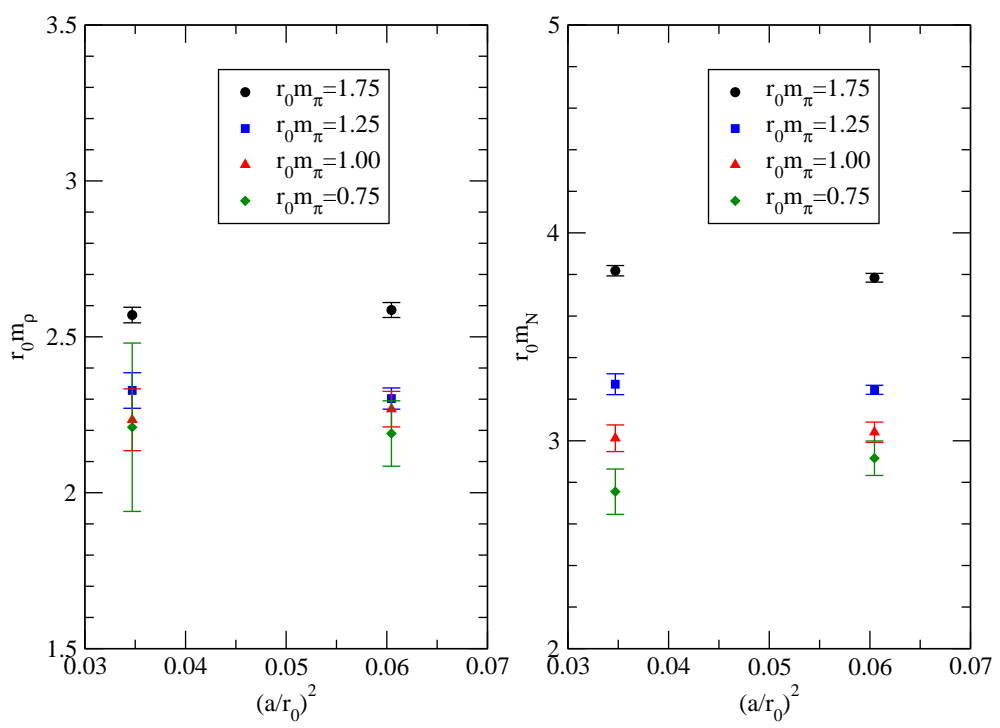

FIG. 14: Vector meson and $(1 / 2)^{+}$baryon masses as functions of lattice spacing squared, calculated using the parity definition of maximal twist: $\omega=\pi / 2$ in Eq. (15). Axes are in units of $r_{0}=0.5$ fm. 

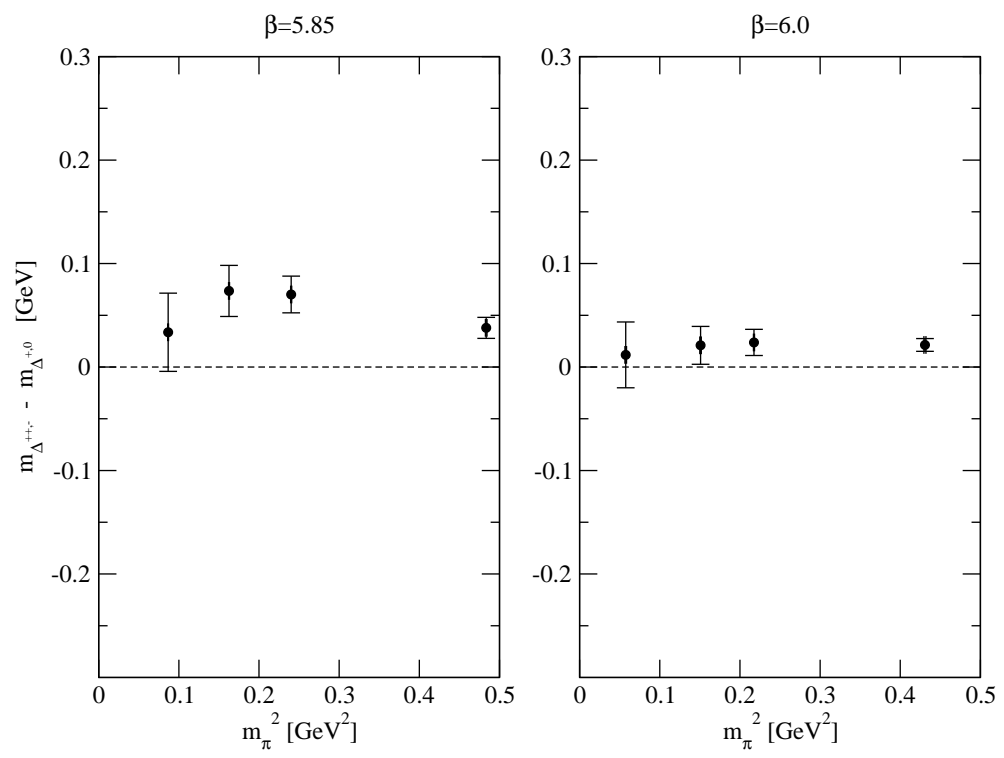

FIG. 15: Flavor splitting within the $\Delta(1232)$ multiplet as a function of the pseudoscalar meson mass squared. The standard mass parameter was held fixed to the value obtained from the Wilson $\kappa_{c W}$ definition of maximal twist, Eq. (12).
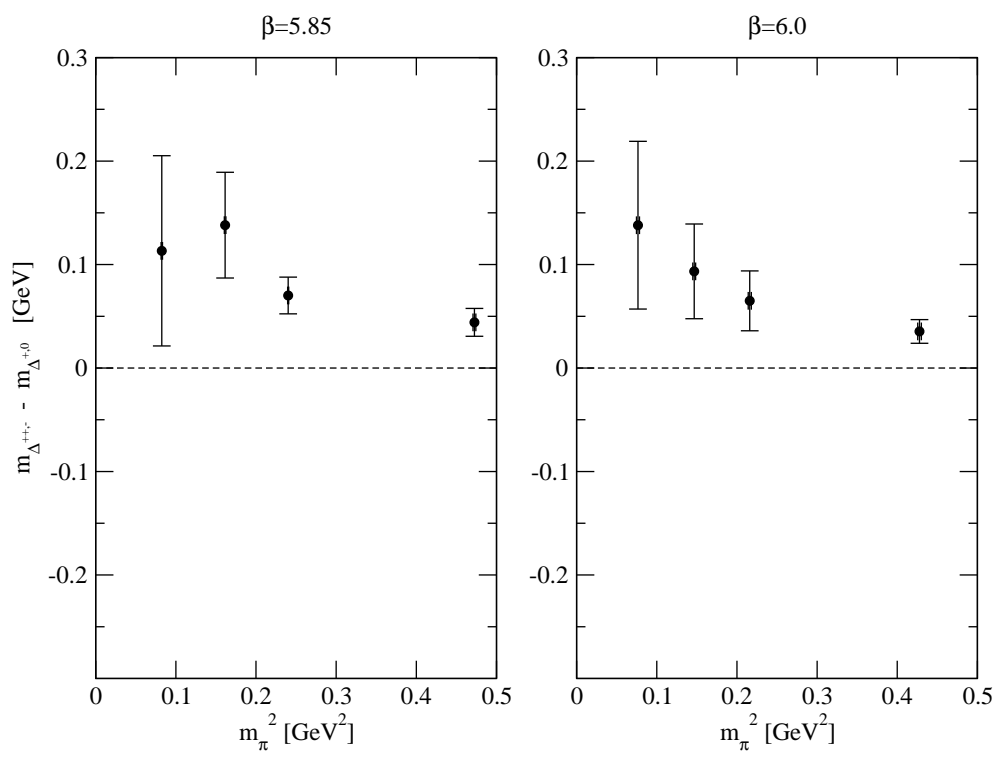

FIG. 16: Flavor splitting within the $\Delta(1232)$ multiplet as a function of the pseudoscalar meson mass squared, calculated using the parity conservation definition of maximal twist: $\omega=\pi / 2$ in Eq. (15). 